\title{
Automatic identification of fossils and abiotic grains during carbonate microfacies analysis using deep convolutional neural networks
}

\author{
Xiaokang Liu', Haijun Song1* \\ ${ }^{1}$ State Key Laboratory of Biogeology and Environmental Geology, School of Earth Sciences, China \\ University of Geosciences, Wuhan 430074, China \\ *Corresponding author. Email: haijunsong@cug.edu.cn
}

\begin{abstract}
Petrographic analysis based on microfacies identification in thin sections is widely used in sedimentary environment interpretation and paleoecological reconstruction. Fossil recognition from microfacies is an essential procedure for petrographers to complete this task. Distinguishing the morphological and microstructural diversity of skeletal fragments requires extensive prior knowledge of fossil morphotypes in microfacies and long training sessions under the microscope. This requirement engenders certain challenges for sedimentologists and paleontologists, especially novices. However, a machine classifier can help address this challenge. In this study, we collected a microfacies image dataset comprising both public data from 1133 references and our own materials (including a total of 30,815 images of 22 fossil and abiotic grain groups). We employed a high-performance workstation to implement four classic deep convolutional neural networks, which
\end{abstract}


have proven to be highly efficient in computer vision. Our framework uses a transfer learning technique, which reuses the pre-trained parameters that are trained on a larger ImageNet dataset as initialization for the network to achieve high accuracy with low computing costs. We obtained up to $95 \%$ of the top one and $99 \%$ of the top three test accuracies in the Inception ResNet v2 architecture. The machine classifier exhibited 0.99 precision on minerals such as dolomite and pyrite. Although it had some difficulty on samples having similar morphologies, such as bivalve, brachiopod, and ostracod, it nevertheless obtained 0.88 precision. Our machine learning framework demonstrates high accuracy with reproducibility and bias avoidance that is comparable to those of human classifiers. Its application can thus eliminate much of the tedious, manually intensive efforts by human experts conducting routine identification.

Keywords: Microfossils, Minerals, Sedimentary structures, Machine learning, Transfer learning

\section{Introduction}

Most petrographic analysis depends on microscopic observation of thin sections. This procedure is typically labor intensive and requires substantial prior knowledge (Wilson, 1975; Flügel, 2010). Most students who examine sedimentary rocks struggle to identify the microfacies, such as the standard microfacies types in Flügel (2010). The most important procedure is to distinguish the morphological and microstructural diversity of skeletal grains. Owing to differences in examiners' abilities and subjective perceptions, identification of fossils and sedimentary structures in thin sections remains challenging for petrographers, especially novices. Meanwhile, 
machine learning algorithms have recently demonstrated high accuracy in image recognition tasks in both computer vision (LeCun et al., 2015; He et al., 2016) and paleontology (Hsiang et al., 2019; Bourel et al., 2020). Hence, we believe that machine learning algorithms can help petrographers to identify fossils during microfacies analysis with reliability and objectivity.

Machine learning has advanced considerably over the last decade (MacLeod et al., 2010; LeCun et al., 2015). This progress is partly attributed to the fact that the use of graphical processing units (GPUs) has significantly increased the speed of training and classification operations in convolutional neural networks (CNNs). The CNN architectures also benefit from GPU acceleration. Hence, scientists have begun to develop deep convolutional neural networks (DCNNs). The ImageNet Large Scale Visual Recognition Challenge (ILSVRC), which contains 1000 categories and over 1.2 million images, has achieved a top-5 error rate of $2.3 \%$ on the validation set in 2017 (Russakovsky et al., 2015). This achievement almost surpasses human identification accuracy. Automatic identification based on machining learning in the fields of sedimentology and paleontology has also been proposed, such as the classification of sedimentary rocks (Shu et al., 2017; Baraboshkin et al., 2020), petrography analysis (Izadi et al., 2017; Shu et al., 2018; DuarteCoronado et al., 2019; Pires de Lima et al., 2019a; 2019b; 2020a), and biotic identification, including planktonic foraminifera (Hsiang et al., 2019; Mitra et al., 2019), fusulinids (Pires de Lima et al., 2020b), pollen grains (Marcos et al., 2015; Bourel et al., 2020), coccoliths (Beaufort and Dollfus, 2004), insects (Larios et al., 2008; Rodner et al., 2015; Valan et al., 2019), benthic invertebrates (Lytle et al., 2010), fish bones and teeth (Hou et al., 2020), and diatoms (Urbankova et al., 2016). 
The early application of neural networks in petrography used composite well data to enable petrophysical identification (Baldwin et al., 1990; Chang et al., 2002; Marmo et al., 2005). With the rise of artificial intelligence and computer vision in the last decade, petrographic analysis studies have diversified into various areas, such as the sorting of particles (Shu et al., 2018), rock classification (Cheng and Guo, 2017; Shu et al., 2017), and microfacies classification (Pires de Lima et al., 2020a). Budennyy et al. (2017) employed machine learning to evaluate the properties of structural objects in thin sections, such as grains, cement, voids, and cleavage. Their models achieved up to $80 \%$ accuracy and proved a way to conduct automatic quantitative and qualitative analysis of thin sections by applying image processing and statistical learning methods. Pires de Lima et al. (2020a) implemented a relatively small number of labeled thin sections (with five classes of rocks from 98 thin sections) used in the fine-tuning method (explained below) and achieved error levels lower than $5 \%$ for the classification of microfacies from the same dataset.

Despite the above efforts, most studies using machine learning employed only local or personal samples materials for convenience. It remains challenging to apply these models for prediction using samples from other regions. For instance, the fossil assemblages of different geological periods may be quite different (Flügel, 2010). Furthermore, some studies used only a limited number of rock types, microfacies classes, or fossil groups, resulting in insufficient actual classification.

Meanwhile, DCNNs require massive training datasets and can thus produce accurate identifications. In this study, we focused on identifying fossil fragments from microfacies. We collected 22 of the most common types of fossil and mineral groups as well as sedimentary 
structures from both the published literature and our own collection. More than 30,000 images were used. We implemented four classic DCNNs that have a high test accuracy.

\section{Materials and data}

In this study, we used both image data collected from publicly available literature and our materials from the Permian and Triassic in South China. A total of 21,839 images (almost all of them from thin sections) were collected from 1133 references (An appendix file provided for original references) from the Phanerozoic Eon; A total of 8976 images were from our samples in South China (Song et al., 2009a, 2009b; Song et al., 2015; Dai et al., 2018; Jia and Song, 2018; Liu et al., 2020, plus additional unpublished materials). The majority of the public data was collected from thin sections reported in the literature. We use Adobe Acrobat DC to crop the separate fossil and abiotic grains from published images. We do not strictly limit the size and resolution of the images, as long as its quality is sufficient for identification. The database ultimately contains 30,815 images of 18 fossil groups as well as four minerals or sedimentary structures: algae, bivalve, brachiopod, bryozoan, calcimicrobe, calcisphere, calpionellid, cephalopod, coral, echinoderm, foraminifer, gastropod, ostracod, radiolarian, sponge, stromatolite, stromatoporoid, Tubiphytes, dolomite, oncolite, ooid, and pyrite (Fig. 1). We randomly divided all data into three categories: training set $(80 \%$ of data), validation set ( $15 \%$ of data), and test set ( $5 \%$ of data). Details are provided in Table 1. The training set was used for model fitting, the validation set was used to tune the model hyperparameters and initially evaluate the model's ability, and the test set was used to evaluate the generalization ability of the final model. 


\section{Methods}

All analysis codes were run on a Dell Precision 7920 Workstation desktop on Windows 10 Professional, including two Intel Xeon Silver 4216 Processors, 128 GB of RAM, and two NVIDIA GeForce GTX 2080Ti GPUs (11 GB for each GPU). The versions of Python and TensorFlow used were 3.6.5 and 1.13.1, respectively. The corresponding versions of the NVIDIA CUDA and cuDNN were 10.0 and 7.4, respectively. The algorithms for the DCNN analyses and model weights are available on GitHub (https://github.com/XiaokangLiuCUG/microfacies_analysis_with_dcnn).

\subsection{Convolutional neural network}

The concept of the artificial neural network is inspired by the biological neural networks that constitute animal brains (Hubel and Wiesel, 1962). Fig. 2A depicts a classic artificial neural network, Visual Geometry Group (VGG)-16 network (Simonyan and Zisserman, 2014). A neuron in an idle state accumulates all the signals it has received until it reaches a certain activation threshold (by using the Heaviside step function as activation function) (McCulloch and Pitts, 1943), which is also known as a perceptron. The basic functioning of a signal neuron or perceptron is shown in Fig. 2E and can be expressed as follows:

$$
Y=\varphi\left(\sum_{i=1}^{n} w_{i} x_{i}+b\right),
$$

where $x_{i}$ is the input value, $Y$ denotes the output result, $b$ represents the bias, $w_{i}$ is the weight, $\mathrm{n}$ is the number of the inputs, and $\varphi$ is the activation function (generally a nonlinear activation function, such as ReLU, sigmoid, and tanh). Further, $Y$ can be the input of the next neural or the 
final output of the neural network and eventually achieves classification or regression objectives.

The convolutional neural network (CNN), a class of deep neural networks, is designed and classically used for image recognition, such as image classification and tagging, object detection, and face detection and recognition (LeCun et al., 2015). CNNs are regularized versions of multilayer perceptrons (composed of the convolutional layer(s) and some other layers), as shown in Fig. 2A. The core concept of the CNN is the convolution operation; that is, each image is composed of a matrix of pixel values (usually with one or three channels represented in a grayscale or RGB model). Then, the sub-portion of the input matrix (the $5 \times 5$ matrix in Fig. $2 \mathrm{~B}$ ) can be placed on the convolution with the filter set (the $2 \times 2$ yellow matrix in Fig. 2B). Scrolling of each filter along the matrix computes the inner product of the same filter and input, and each output of the matrix is a feature map (or activation map, the $4 \times 4$ matrix in Fig. 2B, also shown in Fig. 3). Each feature map is followed by a nonlinear activation function to allow neural networks to learn complex decision boundaries (commonly used $\operatorname{ReLU}, \mathrm{f}(\mathrm{x})=\max (0 ; \mathrm{x})$, where $\mathrm{x}$ is the input data, $\mathrm{f}(\mathrm{x})$ is output.) (Nair and Hinton, 2010). After the convolution and activation operations, a pooling layer (Fig. 2 C) is used to merge semantically similar features into a single feature (i.e., downsampling). The end of the CNN is commonly attached to the fully connected layer (Fig. 2D). That is, each neuron in one layer is connected to each neuron in the next layer. The output of the final fully connected layer is the expected output class. These processes, from input images to final output classifications, are called forward propagation (calculation of the network errors). For predicted images, a softmax layer (green rectangle in Fig. 2D) is added, which is used to generate the true probability vector. 
Backpropagation is a learning algorithm for adjusting the weights during the training process (Wlodarczak, 2019). The weights are usually set randomly when the CNNs are initialized. After each training iteration, the error or loss at the output of the multilayer perceptron is calculated and propagated back through the network to adjust the weights and ensure error minimization in the next iteration. An appropriate optimizer algorithm provides a correct and effective direction to increase the performance of the neural networks. Commonly used optimizers are Adam, RMSprop, and SGD. An iteration is not complete until both forward propagation and backpropagation are completed. Theoretically, an epoch is completed when each image from the training dataset is fit for both forward propagation and backpropagation.

\subsection{Transfer learning}

Deep convolutional neural networks are accompanied by numerous training parameters.

Taking the VGG-16 architecture as an example, there are 138 million parameters trained on the ImageNet dataset with 1.2 million labelled images, including 1000 classes (Deng et al., 2009; Simonyan and Zisserman, 2014; Russakovsky et al., 2015). However, in practice, we usually do not have enough data to newly train such complex DCNN architectures. Therefore, transfer learning is a high-efficiency approach to compensate for dataset insufficiency. We take DCNN's parameters that have already been trained in a larger dataset (such as ImageNet) and make appropriate changes to fit in our smaller dataset. Some layers are frozen (such as Conv1 to Conv5 layers in Fig. 2A, and its parameters will be untrainable) as a feature extractor. Additionally, we used a few trainable layers ( $\mathrm{fc} 6-\mathrm{fc} 8$ layers in Fig. 2A) on the top of the frozen layers as the classifier. Another alternative 
method is fine-tuning, which unfreezes all the layers (or most of the layers) using the pre-trained parameters as initial values and re-training them on our dataset with a very low learning rate. Transfer learning can achieve high accuracy on a small dataset while reducing both the demand for training data and the training time (Tan et al., 2018).

\subsection{Data augmentation}

Data augmentation is an image pre-processing procedure and a means to "enlarge" the dataset.

Data augmentation is effective in preventing overfitting (that is, when there is high accuracy on the training dataset, but much lower accuracy on the validation or test dataset) with a good generalization ability (Wong et al., 2016; Xu et al., 2016). When we use a small dataset to train the DCNNs, we can implement some pre-processing methods to adjust one image into several images to "deceive" the neural network, such as by (1) randomly horizontally and vertically flipping the images, (2) randomly rotating the images, (3) resizing the scale of the images, (4) randomly cropping the images, (5) transforming the pixel matrices (such as by subtracting the mean values), and (6) adjusting the color space of the images (such as brightness and contrast). VGG and ResNet use methods 1, 3, 4, and 5 (Simonyan and Zisserman, 2014; He et al., 2016). Inception uses methods 1, 3, 4, 5, and 6 (Szegedy et al., 2015).

\subsection{Evaluation metrics}

Evaluation metrics (Fig. 4) is a technique for visualizing, organizing, and selecting classifiers based on artificial neural network performance (Fawcett, 2006). It covers several metrics, such as true positive (TP), false positive (FP), true negative (TN), and false negative (FN) while considering 
one of the labels the positive label (which is usually an interest class). Several common metrics are derived from the confusion matrix: accuracy (the ratio of correctly predicted labels to the total observations); precision (the ratio of correctly predicted positive labels to the total predicted positive observations); recall (the ratio of correctly predicted positive labels to all observations in the actual class; and the F1 score (a comprehensive index that is the harmonic mean of the precision and recall). This score considers both false positives and false negatives (Sarkar et al., 2018).

\subsection{Training architectures}

In this study, we trained four classical DCNNs: VGG-16, ResNet v1-152, Inception v4, and Inception ResNet v2 (Fig. 5). These four DCNN architectures were trained on the ImageNet dataset (Russakovsky et al., 2015), and they performed with high accuracy. Considering the computational limitations of the hardware, we randomly fed the DCNNs with an appropriate batch size for each iteration. We ran each DCNN architecture several times to adjust the hyper-parameters, which could not be trained by the networks. All networks ran from 40 to 60 epochs. The training and validating accuracy were the output of one batch size during the training procedure, and the test accuracy was the average result of all images in the test dataset from each of the two training epochs.

The VGG-16 contains approximately 138 million trainable parameters with 16 layers (Simonyan and Zisserman, 2014). The size of the convolutional kernels used in the VGG-16 architecture is $3 \times 3$. The size of the maxpool kernels are of size $2 \times 2$ with a stride of two; The input image size is $224 \times 224 \times 3$. The ResNet v1-152 contains approximately 60 million trainable parameters and 152 layers (Fig. 5A). It uses the method of residual modules and batch normalization 
techniques (He et al., 2016). Each residual block stacks up its input (identity mapping or skip connection) and output (residual mapping) to be the input of the next layer. The identity mappings can prevent the vanishing gradient issue from building even deeper layers. The input image size is the same as the VGG-16. The Inception architecture is called GoogLeNet. The Inception v4 architecture is composed of 148 layers and approximately 43 million trainable parameters (Fig. 5B). The GoogLeNet group devised a new notion known as "blocks of inception" (network in the network) (Lin et al., 2013; Szegedy et al., 2015), whereby it embeds a multiscale feature extractor, such as $1 \times 1,3 \times 3$, and $5 \times 5$ convolutions, within the same module of the network. The input size for this architecture is $299 \times 299 \times 3$. The Inception ResNet v2 architecture is composed of 164 layers and approximately 55 million trainable parameters (Fig. 5C). This network is inspired by ResNet and is a hybridization of Inception and ResNet architectures, and uses residual connections as an alternative to concatenation filters (Szegedy et al., 2016). The input size for this architecture is consistent with Inception $\mathrm{v} 4$.

\section{Results}

\subsection{VGG-16}

The best performance of VGG-16 reached 0.91 for the top one test accuracies and 0.98 for the top three test accuracies (analysis number \#1 in Table 2). The corresponding minimum training and validation losses were 0.12 and 0.52 , respectively (Fig. 6A). In the figure, the losses decline with increased fluctuations, and the accuracy slowly increases. A performance difference occurs between the models trained anew and the fine-tuned models. The former shows 0.6 test accuracy, whereas 
the latter has 0.91 test accuracy. There is a significant bottleneck or barrier of the model that is trained from the beginning. The validation loss starts to increase when the training procedure reaches eight epochs, whereas the training loss seems to decrease effectively. In this situation, with massive parameters, the model is more likely to overfit on the training dataset, which may explain the marked fluctuations in the validation loss of VGG-16 in Fig. 6C. We thus attempted dropout regularization (ranging from 0 to 1.0, where "0" represents probabilistically removing or "dropping out" all the inputs to the next layer and "1" denotes no dropping out) to solve the overfitting (Table 2). The architecture is barely improved when the dropout equals 0.5 , and it reaches the optimal model when the dropout equals 0.8 . The image pre-processing without crop training images (including treatments (1), (3), and (5) in section 3.3) improves approximately $3 \%$ of the test accuracy. VGG-16 identifies 0.87 of the average accuracy in the validation and test datasets. Among them, the prediction precision for calpionellid, radiolarian, pyrite, and dolomite were up to 0.94 , whereas it is more difficult to identify algae (0.69) and bivalves (0.79). The averages of the recall and F1 score were $0.87 \pm 0.07$ and $0.87 \pm 0.06$ respectively. The former ranges from 0.72 to 1 , and the latter ranges from 0.71 to 0.99 (see the Supplementary Table for details).

\subsection{ResNet v1-152}

The optimal performance of ResNet v1-152 recorded 0.94 of the top one test accuracies and 0.99 of the top three test accuracies (analysis number \#7 in Table 2), which was significantly higher than those of VGG-16. The minimum training and validation losses were 0.29 and 0.36 , respectively

(Fig. 6A). The model that trained from the beginning also represented overfitting when training 
stepped up to 20 epochs, and it showed a final test accuracy of 0.68 . ResNet v1-152 also demonstrated accurate performance (0.91 accuracy, analysis number \#8 in Table 2) when the training images were randomly cropped. The dropout function in the last layer had only a slight contribution to model optimization. The average accuracy of the validation and test datasets for analysis number \#7 was 0.89 , and the precision ranged from 0.79 (bivalve) to 1.00 (pyrite). The average F1 score of these 22 classes was $0.89 \pm 0.05$. The highest recall score was for pyrite (1.00), while the lowest for brachiopod obtained 0.79 (also in Supplementary Table).

\subsection{Inception $\mathrm{v} 4$}

Inception v4 exhibited 0.94 of the top one test accuracies and 0.99 of the top three test accuracies (analysis number \#13 in Table 2). The corresponding minimum training and validation losses were 0.19 and 0.28 , respectively (Fig. 6A). Inception $v 4$ also implements batch normalization with good adaptability and quick convergence. The default pre-processing method of the inception architecture uses randomly cropped images, which include a $0.05: 1$ proportion of the original images for network training. The result was approximately $5 \%$ lower than those of the no-crop models (including treatments (1), (3), (5), and (6) in section 3.3). This architecture demonstrated a lower training loss compared with the other three architectures (Fig. 6A). However, the validation loss was inconspicuous, which may be attributed to overfitting or the use of fewer parameters. The final average precision on the validation and test datasets was $0.91 \pm 0.04$. Among them, algae $(0.83)$ and oncolite (0.84) had relatively low precision, whereas radiolarian (0.99) and pyrite (0.99) had high precision. The algae bivalve and sponge contained low recall scores, that is, low sensitivity. 
The averages of recall and $\mathrm{F} 1$ score are $0.92 \pm 0.05$ and $0.91 \pm 0.04$ respectively.

\subsection{Inception ResNet v2}

The Inception ResNet v2 architecture obtained the highest top one test accuracy (0.95) and top three test accuracies (0.99) in all four DCNNs. In this architecture, the minimum training and validation losses were 0.39 and 0.40 , respectively. By combining the advantages of Inception and ResNet networks, Inception ResNet v2 exhibited an effective path for model convergence (Fig. 6D), which was the fastest one to reach the 1.00 validation accuracy (as shown in Fig. 7). In addition, this architecture achieved the highest accuracy in the cropped training images with 0.93 test accuracy (analysis number \#22 in Table 2). The average precision and F1 score for all classes on the validation and test datasets were $0.93 \pm 0.04$. The lowest precision was 0.88 , which was for ostracods (Fig. 8). Dolomite had a precision of 1.00. Classes of bivalve, brachiopod, and oncolite demonstrated $0.87 \mathrm{~F} 1$ scores.

\section{Discussion}

\subsection{Performance evaluation}

We trained four classic DCNNs for image recognition and ran each architecture several times to fine-tune the hyper-parameters. These four architectures showed different performances in fossil classification from the thin sections. Among them, VGG-16 exhibited inferior evaluation performance ( 0.91 of test accuracy), and Inception ResNet v2 obtained the best performance ( 0.95

of test accuracy, model weights are available on 
https://github.com/XiaokangLiuCUG/microfacies_analysis_with_denn). The VGG-16 is more notably affected by overfitting, and its parameters are several times larger than those of other networks. We also attempted to use convolutional layers instead of the fully connected layers (i.e., fc6, cf7, and fc8, which included $86 \%$ of all the parameters) in the posterior three layers. This method partially mitigated the effect of overfitting, and the training and validation loss curves decreased more stably during the 40 training epochs. However, the test accuracy was scarcely improved (0.88).

In addition, we implemented dropout regularization and data augmentation to reduce overfitting on VGG-16, and its effectiveness was distinct. L1/L2 regularization did not improve the validation accuracy on the planktonic foraminifera dataset (Hsiang et al., 2019). A similar overfitting situation occurred on other datasets (Hsiang et al., 2019; Kaya et al., 2019; Mott, 2019). Simonyan and Zisserman (2014) suggested using VGG-16 pre-trained parameters on ILSVRC as feature extractors on other smaller datasets because training large models anew may not be feasible owing to overfitting. Furthermore, in theoretical terms, a deeper network helps the model to extract more features; however, it is more prone to "explode" the gradient (causing it to vanish), resulting in a network's inability or failure to converge (He et al., 2016; Zaccone et al., 2017; Hanin, 2018). Hence, the VGG architecture should use it prudently, especially when the dataset is not large enough for this architecture (Chatfield et al., 2014).

Data augmentation is the most common way to improve the generalization ability of networks in computer vision. Data augmentation is essentially a type of regularization. Using appropriate data 
augmentation methods can improve the generalization ability and accuracy of the model; nevertheless, it will require a longer training time and thus increased costs. In our dataset, the data augmentation technology led to lower accuracies in all architectures in 40-60 training epochs. However, by using a combination of several regularizations, such as dropout, L1/L2 regularization, and early stopping (a technique that monitor the architecture's performance and stop training automatically when the model stopped improving), it could easily lead to underfitting (Zheng et al., 2016; Czyzewski, 2020). With excessive "dropout," the model may have difficulty obtaining sufficient information to continue model optimization, leading to learning bottlenecks. This scenario occurred on the VGG-16 and Inception v4 architectures, in which the validation error barely decreased after 10-15 epochs, whereas Inception ResNet v2 showed greater efficiency (red curves in Fig. 7).

Although underfitting can be compensated by increasing the training epochs or by employing larger batch sizes, the result may still be insignificant (Hsiang et al., 2019). Hence, in terms of the relatively small training time cost, we achieved the best performance evaluation on Inception ResNet v2 by using data augmentation with image cropping. Fine-tuning demonstrates such an efficient and effective approach for learning DCNNs when data are scarce (Sermanet et al., 2013; Wang et al., 2017; Too et al., 2019). We thus conducted several experiments at a low initial learning rate (commonly $10^{-3}$ to $10^{-6}$ ) to unfreeze several or all the layers to investigate the architecture performance. To a certain extent, as the number of activated posterior layers increased, the test accuracy likewise increased (Fig. 7). Activation of shallow convolutional layers did not improve the performances of all four architectures. Only Inception ResNet v2 obtained the highest test accuracy 
by unfreezing all the layers (with 40 training epochs). The shallow convolutional layers that were trained on the larger ImageNet dataset exhibited high performance in detecting low-level features (such as brightness, edges, and curves in Fig. 3) on our dataset. Hence, our results underscore why fine-tuning has been used in many domains (Hentschel et al., 2016; Yin et al., 2017; Kaya et al., 2019).

The DCNNs demonstrated distinctive identification performances on different types of fossils, partially resulting from the intrinsic features of the fossil groups. The fossil or mineral groups have characteristically unique features (such as morphologies and structures) compared to other fossils that could be easily captured by the networks, such as mineral identification (pyrite and dolomite) compared to fossil groups. Here, we implemented t-distributed stochastic neighbor embedding (tSNE) (Maaten and Hinton, 2008) to visualize the high-dimensional features extracted by Inception ResNet v2 (Fig. 9). We plotted 500 images (with an accuracy of 0.94) from the test dataset; among them, 1536 features were extracted from each image after the last Inception ResNet v2 convolutional layer.

By visualizing the extracted features from the DCNN architecture and the confusion matrix (Fig. 8), we noted that, although the machine classifier presented well-identified fossil groups with high generalization ability, it demonstrated ambiguous identification in four major categories: (1) algae versus foraminifer and sponge; (2) bivalve, brachiopod, and ostracod; (3) oncolite versus ooid and Tubiphytes; and (4) gastropod versus foraminifer and cephalopod. On the one hand, the misidentification between different classes with similar morphological features could imply the 
inherent difficulty that artificial neural networks have for classes in categories 2 and 3. Images from thin sections could contain random directions of the fossils, which leads to more fickle characteristic for each class. Although our data contains both greyscale and color images, the machine classifier demonstrates barely any difference of the performance applied to these images. We therefore transferred the greyscale images into "false" color matrixes for the input of the CNN architecture, and the neutral networks could capture the features when we provide enough training examples. Background noise also could influence the performance of the algorithms. In the 22 classes we include here, some of the groups are composed of plain morphologies, such as bivalve, ostracod, calcisphere, and calpionellid. The architectures could misidentify when the algorithms capture the background noise rather the true features of the fossils. Collecting more data and with appropriate image pre-processing methods could partially compensate for these deficiencies (Russakovsky et al., 2015). On the other hand, the composition of the dataset affects the performance of the machine classifier. In our collection, although more than two-thirds of our data was from the literature, several fossil groups remained insufficient, such as brachiopod, cephalopod, gastropod, radiolarian, and Tubiphytes. Therefore, we supplemented it with our materials from the Permian to Triassic in South China. Nevertheless, it was still difficult for us to distribute our data relatively evenly spread in high taxonomic groups for some classes (i.e., within-class imbalanced data), considering the wide morphological variation between high taxonomic groups in one fossil group, such as algae, foraminifers, and sponges. This deficiency resulted in identification biases within some classes, such as a higher accuracy on green algae compared to red algae (both identified as algae), a higher accuracy on crinoids compared to echinoids (both identified as echinoderm), and a higher accuracy 
on spiral-shelled cephalopods compared to straight-shelled cephalopods. Another within-dataset imbalanced situation is that the proportion of data from a few sources in the database is significantly higher than other sources. This could elevate the performance of the models during the experimental stage due to the higher similarities in the matrixes, background textures, and taphonomic regimes, but poorly perform in the real open database.

Hsiang et al. (2019) reported that a single class with a higher representation (abundance) leads to identification bias, which also results in decreased accuracy for undersampled foraminiferal species. There are several methods to partially compensate for imbalanced data (Chawla et al., 2002; Batista et al., 2004; He and Garcia, 2009; Krawczyk, 2016). The sampling method is the most common method by random oversampling of classes with minority data and undersampling the classes with majority data. Other methods, such as cost-sensitive methods, kernel-based methods, and active learning methods, are also frequently implemented to improve imbalanced datasets (He and Garcia, 2009). In our case, we removed some data from the classes (i.e., radiolarian, coral, and foraminifer) that had higher quantities to prevent highly skewed datasets.

Compared with human identification, the machine classifier works without prior experience and conducts identification based on the recognized image characteristics from the training phase. This implies that the CNN architecture can only identify the classes that have been previously trained. Any new class of images will still generate a probability vector belonging to those in classes that have been trained, as in the experimental studies of Pires de Lima et al. (2020a). Moreover, the machine classifier has the potential advantage of having high accuracy, reproducibility, and bias 
avoidance (Hsiang et al., 2019). In practice, the human classifier is common with accurate identification of several common taxa (having a higher occurrence) and poor identification of rare species. Even the accuracies of human experts are highly dependent on individual performance, often in unpredictable ways (Hsiang et al., 2019). Austen et al. (2018) conducted an investigation in which 17 experts were invited to identify four newt species from websites. The researchers found that additional years of experience did not improve the experts' identification performances; rather, increased expertise could result in a participant being more cautious in performing identification (Austen et al., 2016). Similarly, Al-Sabouni et al. (2018) determined that the length of an expert's experience did not correlate with higher identification accuracy for planktonic foraminifers. Meanwhile, taxonomists self-trained by reading books were more likely to exhibit lower accuracies with more divergent opinions compared to the community consensus. On the other hand, some of the species may only be reliably identified by a few core experts in the field but commonly misidentified by most practitioners (Hsiang et al., 2019). For example, Hsiang et al. (2019) noticed that a majority of images classified as Globigerina bulloides should actually be identified as Globigerina falconensis. The latter contains much rarer abundance and is unfamiliar even for some expert taxonomists. However, the performance of machine classifier mainly depends on inherent properties such as algorithms and original dataset. Another difference between human experts and machine classifiers is that human classifiers tend to be more phylogenetically conservative in their mistakes (Hsiang et al., 2019), i.e., the misidentified images commonly occur at the correct genus level. The machine classifier has mistaken identifications that occur in morphological similarity or even more random in all classes. In summary, the machine classifier now can reproduce instantly 
and objectively some recognition objectives that humans have already achieved. However, the means of addressing these results still depends on human experts (MacLeod et al., 2010). The CNN architectures also suffered from imbalanced data as discussed above, but less impacted compared with human classifiers (Hsiang et al., 2019). Hsiang et al. (2019) suggested that larger and unskewed samples can lead to more robust models, which can in turn result in a higher quality of machine accuracies that is comparable to (or may even surpass) human classifiers.

\subsection{Machine learning in petrography and paleontology}

Artificial neural networks in the microfacies classification that is based on petrographic thin sections have provided performances comparable to that of a petrographer. Previous studies have employed microfacies classification in both clastic sediments and carbonate rocks (Marmo et al., 2005; Pires de Lima et al., 2020a). Pires de Lima et al. (2020a) used the DCNN fine-tuning method to distinguish five similar siltstones, including argillaceous, bioturbated, and porous calcareous siltstone. Although their model can adapt DCNNs to achieve low error levels $(<5 \%)$, their data were constrained by numerous sub-images, which were generated from a larger size of the original image. This led to the CNN classification having a higher accuracy for the particular sub-image during the analysis, whereas it engendered a lower generalization ability on other new images (Pires de Lima et al., 2020a). Another notable issue is that some of their training datasets contained images that were stained for specific minerals, such as calcite or dolomite identification; however, normally public data were missing those stained features that generalization ability would greatly reduce

(Pires de Lima et al., 2020a). In further research, scholars can implement machine learning in semi- 
quantitative analysis of abstract features, such as permeability, porosity, and sorting levels of rock particles. This approach is somewhat subjective for human classifiers, especially for novices. These comprehensive structures can be divided into different sorting levels, such as very well sorted, moderately sorted, and poorly sorted (Shu et al., 2018), and different porosity levels, such as low, medium, and high porosity (Duarte-Coronado et al., 2019). In this circumstance, the petrographer can discretely and objectively label the porosity or sorting level of the training dataset to reduce interpretation bias. This can engender another significant issue with respect to biases due to the image. Thus, when addressing thin section heterogeneities, researchers should consider using the appropriate scale or size of the images for machine learning. The pre-processing used for the image cropping and scale resizing methods discussed previously is also worthy of caution. An example was demonstrated by Pires de Lima et al. (2020a), who found that the identification of level of bioturbations in thin sections were influenced by the scale of training images. Sometimes the bioturbation evidence is obscured when cropping the thin section images into smaller $10 \times$ magnification images.

Compared with previous studies on petrographic microfacies classifications, the present study is the first to focus on the identification of 18 common fossil group fragments which collected in thin sections from the Phanerozoic Eon. More than two-thirds of the data is from the literature, which provides our models with excellent generalization ability for both newly acquired data and our own samples. High taxonomic fossils groups (or even genera to species) identification in carbonate skeletons is essential for sedimentary environment analyses. In fact, our method is a semiautomatic identification method, which requires the preparation of a single fossil image for 
prediction. Subsequent research can be carried out from a real-time object detection task to implement the identification of multiple classes and fossils from a single microfacies image. Ultimately, fully automated carbonate petrography could be used to identify the fossils of the images captured from the camera in the microscope.

Although the earliest artificial intelligence techniques in biometric identification research have been employed since the 1970s, only a minimal amount of exploratory research has been conducted in the last several decades, mostly on invertebrates and microfossils (Pankhurst, 1974; MacLeod, 2007). Considering the costs of time, human resources, and financial resources, only a few segments of research can develop into continuous achievements to create useful popularization tools for biologists or paleontologists (MacLeod et al., 2010). Nonetheless, benefits have been provided by the rapid development of computer science in the past decade (Krizhevsky et al., 2012; Chetlur et al., 2014; Schmidhuber, 2015; Abadi et al., 2016), and many geological datasets are being digitized and standardized for sharing with peers, such as through publications and online databases (Hsiang et al., 2019). By collecting both online and offline geological big data and applying them for artificial intelligence use, human experts can be alleviated of the tedium of conducting routine identifications. For instance, we are striving to implement the microfacies automatic identification model that we trained in the present study in an online database for all petrographers and students. This will provide a convenient and efficient means for performing fossil identification during microfacies analysis. 


\section{Conclusions}

In this study, a deep learning algorithm that uses digital images to automatically classify fossil and abiotic grains from thin sections was implemented. Four classic DCNN architectures were trained on 30,815 images, which included 22 classes. All architectures obtained higher than 0.9 accuracies. Among them, the optimal model was demonstrated in the Inception ResNet v2 architecture. It exhibited $95 \%$ of the top one and $99 \%$ of the top three test accuracies. By using public data and our materials for model training, the architectures were more robust and had an improved generalization ability. Transfer learning is a powerful technology for implementing complex DCNN architectures in a relatively small dataset. The machine classifier performance was affected by data quality, data augmentation, and hyper-parameters; however, it showed the advantages of rapid identification, reproducibility, and bias avoidance compared with human classifiers. Automatic identification in petrography and paleontology based on DCNNs can provide geologists with an alternative and convenient method for routine and labor-intensive identification tasks.

\section{Acknowledgements}

We thank Yongbiao Wang, Jing Chen, and Zheng Meng for supplying data, Minghui Wu, Zhuorong Li and Baichuan Jin for help on algorithm improvements, Shouyi Jiang for supporting hardware equipment in the early stage. We would also like to thank two anonymous reviewers for their insightful comments. This study is supported by the National Natural Science Foundation of China (41821001), the State Key R\&D Project of China (2016YFA0601100), and Strategic Priority 
Research Program of Chinese Academy of Sciences (XDB26000000). This is Center for Computational \& Modeling Geosciences publication number 1.

\section{References}

Abadi, M., Barham, P., Chen, J., Chen, Z., Davis, A., Dean, J., Devin, M., Ghemawat, S., Irving, G., Isard, M., 2016. Tensorflow: A system for large-scale machine learning, 12th USENIX symposium on operating systems design and implementation, USENIX Association, Savannah, USA, pp. 265-283.

Akgümüss, B., 2019. Taxonomy and biostratigraphy of calpionellids and saccocoma across the Jurassic-Cretaceous boundary beds of the Alci Block: Alacaatli olistostromes (Ph.D. thesis). Middle East Technical University, Ankara, Turkey, 243pp.

Al-Sabouni, N., Fenton, I.S., Telford, R.J., Kucera, M., 2018. Reproducibility of species recognition in modern planktonic foraminifera and its implications for analyses of community structure. Journal of Micropalaeontology 37, 519-534.

Austen, G.E., Bindemann, M., Griffiths, R.A., Roberts, D.L., 2016. Species identification by experts and non-experts: comparing images from field guides. Scientific Reports 6, 33634 . https://doi.org/10.1038/srep33634.

Austen, G.E., Bindemann, M., Griffiths, R.A., Roberts, D.L., 2018. Species identification by conservation practitioners using online images: accuracy and agreement between experts. PeerJ 6, e4157. https://doi.org/10.7717/peerj.4157.

Baldwin, J.L., Bateman, R.M., Wheatley, C.L., 1990. Application of A Neural Network to the 
Problem of Mineral Identification from Well Logs. The Log Analyst 31, 279-293.

Baraboshkin, E.E., Ismailova, L.S., Orlov, D.M., Zhukovskaya, E.A., Kalmykov, G.A., Khotylev, O.V., Baraboshkin, E.Y., Koroteev, D.A., 2020. Deep convolutions for in-depth automated rock typing. Computers \& Geosciences $135, \quad 104330$. https://doi.org/10.1016/j.cageo.2019.104330.

Batista, G.E., Prati, R.C., Monard, M.C., 2004. A study of the behavior of several methods for balancing machine learning training data. ACM SIGKDD Explorations Newsletter 6, 2029.

Beaufort, L., Dollfus, D., 2004. Automatic recognition of coccoliths by dynamical neural networks. Marine Micropaleontology 51, 57-73.

Bourel, B, Marchant, R., de Garidel-Thoron, T., Tetard, M., Barboni, D., Gally, Y., Beaufort, L., 2020. Automated recognition by multiple convolutional neural networks of modern, fossil, intact and damaged pollen grains. Computers \& Geosciences 140, 104498. https://doi.org/10.1016/j.cageo.2020.104498.

Budennyy, S., Pachezhertsev, A., Bukharev, A., Erofeev, A., Mitrushkin, D., Belozerov, B., 2017. Image Processing and Machine Learning Approaches for Petrographic Thin Section Analysis. SPE Russian Petroleum Technology Conference, Society of Petroleum Engineers, Moscow, Russia, pp. 1-12.

Chang, H.-C., Kopaska-Merkel, D.C., Chen, H.-C., 2002. Identification of lithofacies using Kohonen self-organizing maps. Computers \& Geosciences 28, 223-229.

Chatfield, K., Simonyan, K., Vedaldi, A., Zisserman, A., 2014. Return of the devil in the details: 
Delving deep into convolutional nets. arXiv, 1405.3531.

Chawla, N.V., Bowyer, K.W., Hall, L.O., Kegelmeyer, W.P., 2002. SMOTE: synthetic minority over-sampling technique. Journal of Artificial Intelligence Research 16, 321-357.

Cheng, G., Guo, W., 2017. Rock images classification by using deep convolution neural network, Journal of Physics: Conference Series887, 012089. https://doi :10.1088/1742$6596 / 887 / 1 / 012089$

Chetlur, S., Woolley, C., Vandermersch, P., Cohen, J., Tran, J., Catanzaro, B., Shelhamer, E., 2014. cudnn: Efficient primitives for deep learning. arXiv, 1410.0759.

Czyzewski, M.A., 2020. batchboost: regularization for stabilizing training with resistance to underfitting \& overfitting. arXiv, 2001.07627.

Dai, X., Song, H., Wignall, P.B., Jia, E., Bai, R., Wang, F., Chen, J., Tian, L., 2018. Rapid biotic rebound during the late Griesbachian indicates heterogeneous recovery patterns after the Permian-Triassic mass extinction. GSA Bulletin 130, 2015-2030.

Deng, J., Dong, W., Socher, R., Li, L., Li, K., Li, F., 2009. Imagenet: A large-scale hierarchical image database. 2009 IEEE Conference on Computer Vision and Pattern Recognition. IEEE, Miami, USA, pp. 248-255.

Donatelli, U., Tramontana, M., 2014. Platform-to-basin facies transition and tectono-sedimentary processes in the Jurassic deposits of the Furlo area (Umbria-Marche Apennines, Italy). Facies 60, 541-560.

Duarte-Coronado, D., Tellez-Rodriguez, J., Pires de Lima, R., Marfurt, K., Slatt, R., 2019. Deep convolutional neural networks as an estimator of porosity in thin-section images for 
unconventional reservoirs. SEG International Exposition and 89th Annual Meeting. Society of Exploration Geophysicists, San Antonio, USA, pp. 3181-3184.

Fawcett, T., 2006. An introduction to ROC analysis. Pattern Recognition Letters 27, 861-874.

Flügel, E., 2010. Microfacies of Carbonate Rocks: analysis, interpretation and application 2nd Edition. Springer, Berlin, Heidelberg, 976 pp.

Franchi, F., Turetta, C., Cavalazzi, B., Corami, F., Barbieri, R., 2016. Trace elements and REE geochemistry of Middle Devonian carbonate mounds (Maïder Basin, Eastern Anti-Atlas, Morocco): Implications for early diagenetic processes. Sedimentary Geology 343, 56-71.

Ghavidel-Syooki, M., 2017. Stratigraphic evidence for Hirnantian glaciation in the Alborz Mountain Ranges, northeastern Iran. Palaeogeography, Palaeoclimatology, Palaeoecology 485, 879-898.

Grosjean, A.-S., Vennin, E., Olivier, N., Caravaca, G., Thomazo, C., Fara, E., Escarguel, G., Bylund, K.G., Jenks, J.F., Stephen, D.A., 2018. Early Triassic environmental dynamics and microbial development during the Smithian-Spathian transition (Lower Weber Canyon, Utah, USA). Sedimentary Geology 363, 136-151.

Gu, J., Wang, Z., Kuen, J., Ma, L., Shahroudy, A., Shuai, B., Liu, T., Wang, X., Wang, G., Cai, J., 2018. Recent advances in convolutional neural networks. Pattern Recognition 77, 354-377.

Hanin, B., 2018. Which neural net architectures give rise to exploding and vanishing gradients? Advances in Neural Information Processing Systems. Curran Associates, Inc. Montréal Canada, pp. 582-591.

He, H., Garcia, E.A., 2009. Learning from imbalanced data. IEEE Transactions on Knowledge and 
Data Engineering 21, 1263-1284.

He, K., Zhang, X., Ren, S., Sun, J., 2016. Identity mappings in deep residual networks. Computer Vision-ECCV 2016. Springer International Publishing, Cham, pp. 630-645.

Hentschel, C., Wiradarma, T.P., Sack, H., 2016. Fine tuning CNNS with scarce training dataAdapting ImageNet to art epoch classification. 2016 IEEE International Conference on Image Processing (ICIP). IEEE, Phoenix, USA, pp. 3693-3697.

Hou, Y., Cui, X., Canul-Ku, M., Jin, S., Hasimoto-Beltran, R., Guo, Q., Zhu, M., 2020. ADMorph: A 3D Digital Microfossil Morphology Dataset for Deep Learning. IEEE Access 8, 148744148756.

Hsiang, A.Y., Brombacher, A., Rillo, M.C., Mleneck - Vautravers, M.J., Conn, S., Lordsmith, S., Jentzen, A., Henehan, M.J., Metcalfe, B., Fenton, I.S., 2019. Endless Forams:> 34,000 modern planktonic foraminiferal images for taxonomic training and automated species recognition using convolutional neural networks. Paleoceanography and Paleoclimatology $34,1157-1177$.

Hubel, D.H., Wiesel, T.N., 1962. Receptive fields, binocular interaction and functional architecture in the cat's visual cortex. The Journal of Physiology 160, 106-154.

Izadi, H., Sadri, J., Bayati, M., 2017. An intelligent system for mineral identification in thin sections based on a cascade approach. Computers \& Geosciences 99, 37-49.

Jia, E., Song, H., 2018. End-Permian mass extinction of calcareous algae and microproblematica from Liangfengya, South China. Geobios 51, 401-418.

Kaya, A., Keceli, A.S., Catal, C., Yalic, H.Y., Temucin, H., Tekinerdogan, B., 2019. Analysis of 
transfer learning for deep neural network based plant classification models. Computers and Electronics in Agriculture 158, 20-29.

Kröger, B., Penny, A., Shen, Y., Munnecke, A., 2020. Algae, calcitarchs and the Late Ordovician Baltic limestone facies of the Baltic Basin. Facies 66, 1. https://doi.org/10.1007/s10347019-0585-0

Krawczyk, B., 2016. Learning from imbalanced data: open challenges and future directions. Progress in Artificial Intelligence 5, 221-232.

Krizhevsky, A., Sutskever, I., Hinton, G.E., 2012. Imagenet classification with deep convolutional neural networks, Advances in neural information processing systems, Curran Associates, Inc. Lake Tahoe, USA, pp. 1097-1105.

Lakew, T., 1990. Microfacies and cyclic sedimentation of the Upper Triassic (Rhaetian) Calcare di $\mathrm{Zu}$ (southern Alps). Facies 22, 187-231.

Larios, N., Deng, H., Zhang, W., Sarpola, M., Yuen, J., Paasch, R., Moldenke, A., Lytle, D.A., Correa, S.R., Mortensen, E.N., 2008. Automated insect identification through concatenated histograms of local appearance features: feature vector generation and region detection for deformable objects. Machine Vision and Applications 19, 105-123.

LeCun, Y., Bengio, Y., Hinton, G., 2015. Deep learning. Nature 521, 436-444.

Lin, M., Chen, Q., Yan, S., 2013. Network in network. arXiv, 1312.4400.

Liu, X., Song, H., Bond, D.P., Tong, J., Benton, M.J., 2020. Migration controls extinction and survival patterns of foraminifers during the Permian-Triassic crisis in South China. EarthScience Reviews 209, 103329. https://doi.org/10.1016/j.earscirev.2020.103329 
Lytle, D.A., Martínez-Muñoz, G., Zhang, W., Larios, N., Shapiro, L., Paasch, R., Moldenke, A., Mortensen, E.N., Todorovic, S., Dietterich, T.G., 2010. Automated processing and identification of benthic invertebrate samples. Journal of the North American Benthological Society $29,867-874$.

Maaten, L.v.d., Hinton, G., 2008. Visualizing data using t-SNE. Journal of Machine Learning Research 9, 2579-2605.

MacLeod, N., 2007. Automated taxon identification in systematics: theory, approaches and applications. CRC Press, Boca Raton, 339 pp.

MacLeod, N., Benfield, M., Culverhouse, P., 2010. Time to automate identification. Nature 467, $154-155$.

Marcos, J.V., Nava, R., Cristóbal, G., Redondo, R., Escalante-Ramírez, B., Bueno, G., Déniz, Ó., González-Porto, A., Pardo, C., Chung, F., 2015. Automated pollen identification using microscopic imaging and texture analysis. Micron 68, 36-46.

Marmo, R., Amodio, S., Tagliaferri, R., Ferreri, V., Longo, G., 2005. Textural identification of carbonate rocks by image processing and neural network: Methodology proposal and examples. Computers \& Geosciences 31, 649-659.

Martin-Bello, L., Arenas, C., Jones, B., 2019. Lacustrine stromatolites: Useful structures for environmental interpretation-an example from the Miocene Ebro Basin. Sedimentology 66, 2098-2133.

McCulloch, W.S., Pitts, W., 1943. A logical calculus of the ideas immanent in nervous activity. The Bulletin of Mathematical Biophysics 5, 115-133. 
McLean, R.A., 2005. Phillipsastreid corals from the Frasnian (Upper Devonian) of Western Canada: Taxonomy and biostratigraphic significance. NRC Research Press, Ottawa, Canada, 109 pp.

Mitra, R., Marchitto, T., Ge, Q., Zhong, B., Kanakiya, B., Cook, M., Fehrenbacher, J., Ortiz, J., Tripati, A., Lobaton, E., 2019. Automated species-level identification of planktic foraminifera using convolutional neural networks, with comparison to human performance. Marine Micropaleontology 147, 16-24.

Mott, K., 2019. State Classification of Cooking Objects Using a VGG CNN. arXiv: 1904.12613.

Nair, V., Hinton, G.E., 2010. Rectified linear units improve restricted boltzmann machines. 27th International Conference on Machine Learning. ICML, Haifa, Israel, pp. 807-814.

Pankhurst, R.J., 1974. Automated identification in systematics. Taxon 23, 45-51.

Pires de Lima, R., Bonar, A., Coronado, D.D., Marfurt, K., Nicholson, C., 2019a. Deep convolutional neural networks as a geological image classification tool. Sedimentary Record 17, 4-9.

Pires de Lima, R., Suriamin, F., Marfurt, K.J., Pranter, M.J., 2019b. Convolutional neural networks as aid in core lithofacies classification. Interpretation 7, SF27-SF40.

Pires de Lima, R., Duarte, D., Nicholson, C., Slatt, R., Marfurt, K.J., 2020a. Petrographic microfacies classification with deep convolutional neural networks. Computers \& Geosciences 142, 104481. https://doi.org/10.1016/j.cageo.2020.104481.

Pires de Lima, R., Welch, K.F., Barrick, J.E., Marfurt, K.J., Burkhalter, R., Cassel, M., Soreghan, G.S., 2020b. Convolutional neural networks as an aid to biostratigraphy and 
micropaleontology: a test on Late Paleozoic microfossils. Palaios 35, 391-402.

Rodner, E., Simon, M., Brehm, G., Pietsch, S., Wägele, J.W., Denzler, J., 2015. Fine-grained recognition datasets for biodiversity analysis. arXiv, 1507.00913.

Russakovsky, O., Deng, J., Su, H., Krause, J., Satheesh, S., Ma, S., Huang, Z., Karpathy, A., Khosla, A., Bernstein, M., 2015. Imagenet large scale visual recognition challenge. International Journal of Computer Vision 115, 211-252.

Salama, W., El Aref, M., Gaupp, R., 2014. Facies analysis and palaeoclimatic significance of ironstones formed during the Eocene greenhouse. Sedimentology 61, 1594-1624.

Sarkar, D., Bali, R., Ghosh, T., 2018. Hands-On Transfer Learning with Python: Implement advanced deep learning and neural network models using TensorFlow and Keras. Packt Publishing Ltd., Birmingham, UK, 438 pp.

Sartorio, D., Venturini, S., 1988. Southern tethys biofacies. AGIP Press, Italy, 235 pp.

Schlagintweit, F., 2008. Bioerosional structures and pseudoborings from Late Jurassic and Late Cretaceous-Paleocene shallow-water carbonates (Northern Calcareous Alps, Austria and SE France) with special reference to cryptobiotic foraminifera. Facies54, 377-402.

Schmidhuber, J., 2015. Deep learning in neural networks: An overview. Neural Networks 61, 85117.

Scholle, P.A., Ulmer-Scholle, D.S., 2003. A Color Guide to the Petrography of Carbonate Rocks: Grains, Textures, Porosity, Diagenesis, 77. American Association of Petroleum Geologists, Tulsa, Oklahoma, $474 \mathrm{pp}$.

Senowbari-Daryan, B., 2013. Tubiphytes Maslov, 1956 and description of similar organisms from 
Triassic reefs of the Tethys. Facies 59, 75-112.

Sermanet, P., Eigen, D., Zhang, X., Mathieu, M., Fergus, R., LeCun, Y., 2013. Overfeat: Integrated recognition, localization and detection using convolutional networks. arXiv,1312.6229.

Shu, L., McIsaac, K., Osinski, G.R., Francis, R., 2017. Unsupervised feature learning for autonomous rock image classification. Computers \& Geosciences 106, 10-17.

Shu, L., Osinski, G.R., McIsaac, K., Wang, D., 2018. An automatic methodology for analyzing sorting level of rock particles. Computers \& Geosciences 120, 97-104.

Simonyan, K., Zisserman, A., 2014. Very deep convolutional networks for large-scale image recognition. arXiv, 1409.1556 .

Song, H., Tong, J., Chen, Z., 2009a. Two episodes of foraminiferal extinction near the PermianTriassic boundary at the Meishan section, South China. Australian Journal of Earth Sciences 56, 765-773.

Song, H., Tong, J., Chen, Z., Yang, H., Wang, Y., 2009b. End-Permian mass extinction of foraminifers in the Nanpanjiang Basin, South China. Journal of Paleontology 83, 718-738.

Song, H., Yang, L., Tong, J., Chen, J., Tian, L., Song, H., Chu, D., 2015. Recovery dynamics of foraminifers and algae following the Permian-Triassic extinction in Qingyan, South China. Geobios 48, 71-83.

Szegedy, C., Ioffe, S., Vanhoucke, V., Alemi, A., 2016. Inception-v4, inception-resnet and the impact of residual connections on learning. arXiv, 1602.07261.

Szegedy, C., Liu, W., Jia, Y., Sermanet, P., Reed, S., Anguelov, D., Erhan, D., Vanhoucke, V., Rabinovich, A., 2015. Going deeper with convolutions. Proceedings of the IEEE 
Conference on Computer Vision and Pattern Recognition. IEEE, Boston, USA, pp. 1-9.

Tan, C., Sun, F., Kong, T., Zhang, W., Yang, C., Liu, C., 2018. A Survey on Deep Transfer Learning. Artificial Neural Networks and Machine Learning-ICANN 2018. Springer International Publishing, Cham, pp. 270-279.

Too, E.C., Yujian, L., Njuki, S., Yingchun, L., 2019. A comparative study of fine-tuning deep learning models for plant disease identification. Computers and Electronics in Agriculture $161,272-279$

Urbankova, P., Scharfen, V., Kulichová, J., 2016. Molecular and automated identification of the diatom genus Frustulia in northern Europe. Diatom Research 31, 217-229.

Valan, M., Makonyi, K., Maki, A., Vondráček, D., Ronquist, F., 2019. Automated taxonomic identification of insects with expert-level accuracy using effective feature transfer from convolutional networks. Systematic Biology 68, 876-895.

Wang, Y.-X., Ramanan, D., Hebert, M., 2017. Growing a brain: Fine-tuning by increasing model capacity. Proceedings of the IEEE Conference on Computer Vision and Pattern Recognition, IEEE, Hawaii, USA, pp. 2471-2480.

Wilson, J., 1975. Carbonate facies in geologic history. Springer-Verlag, Berlin, 471 pp.

Wlodarczak, P., 2019. Machine Learning and Its Applications. CRC Press, Florida, USA, 188pp.

Wong, S.C., Gatt, A., Stamatescu, V., McDonnell, M.D., 2016. Understanding data augmentation for classification: when to warp? 2016 international conference on digital image computing: techniques and applications (DICTA). IEEE, Australia, pp. 1-6.

Xu, Y., Jia, R., Mou, L., Li, G., Chen, Y., Lu, Y., Jin, Z., 2016. Improved relation classification by 
deep recurrent neural networks with data augmentation. arXiv, 1601.03651.

Yin, X., Chen, W., Wu, X., Yue, H., 2017. Fine-tuning and visualization of convolutional neural networks, 12th IEEE Conference on Industrial Electronics and Applications (ICIEA). IEEE, Siem Reap, Cambodia, pp. 1310-1315.

Zaccone, G., Karim, M.R., Menshawy, A., 2017. Deep Learning with TensorFlow. Packt Publishing Ltd., Birmingham, UK, 320 pp.

Zheng, S., Song, Y., Leung, T., Goodfellow, I., 2016. Improving the robustness of deep neural networks via stability training. Proceedings of the IEEE Conference on Computer Vision and Pattern Recognition. IEEE, Las Vegas, USA, pp. 4480-4488. 


\section{Figure and table captions}

Fig. 1. Example illustrations of each class in our dataset. Images of algae, brachiopod, bryozoan, gastropod, pyrite, and radiolarian are our own materials. Other photos are from the literature, including bivalve (Grosjean et al., 2018), calcimicrobe (Scholle and Ulmer-Scholle, 2003), calcisphere (Kröger et al., 2020), calpionellid (Akgümüş, 2019), cephalopod (Lakew, 1990), coral (McLean, 2005), dolomite (Franchi et al., 2016), echinoderm (Ghavidel-Syooki, 2017), foraminifer (Sartorio and Venturini, 1988), oncolite (Salama et al., 2014), ooid (Schlagintweit, 2008), ostracod (Donatelli and Tramontana, 2014), sponge and stromatoporoid (Flügel, 2010), stromatolite (MartinBello et al., 2019), and Tubiphytes (Senowbari-Daryan, 2013). Scale(s) for calpionellid is $0.05 \mathrm{~mm}$, for calcisphere dolomite, and radiolarian are $0.1 \mathrm{~mm}$, and for the others are $0.5 \mathrm{~mm}$.

Fig. 2. Schematic of a convolutional neural network and its procedures. (A) A typical architecture of Visual Geometry Group (VGG)-16 network (Simonyan and Zisserman, 2014). It contains 13 convolutional layers, i.e., the blue rectangles; 5 max-pooling layers, i.e., the red rectangles; and three fully connected layers, i.e., purple rectangles. The last green rectangle is the softmax layer, which is the final output classes and generates the probability vector. (B) Schematic of a convolutional operation. Each image can be represented by a pixel matrix, its shape is height $\times$ width $\times$ channels. Here, we demonstrated a $5 \mathrm{px} \times 5 \mathrm{px}$ matrix as the input image, a $2 \times 2$ matrix of filter (yellow rectangle) will scroll (from the left-to-right, top-to-bottom, stride $=1$ ) in the input matrix and computes the inner product with the overlap matrix (brown rectangle), the results will generate a new matrix, also called feature map or activation map. (C) A max-pooling operation with 
stride equals 2 . The adjacent pixels have similar structures, pooling layer aims to downsampling. (D) Fully connected layers in the top of the VGG-16. Every neuron in one layer will connect with every neuron in the next layer. (E) Schematic of an artificial neuron.

Fig. 3. Visualization of the feature maps and heatmaps from different VGG-16 convolutional layers. The heatmaps are fusion maps after the conv5 layers. The shallower layers detected low-level features such as brightness, edges, and curves, while the deeper layers extract more abstract features. By stacking several convolutional and pooling layers, we could gradually encode higher-level feature representations (Gu et al., 2018).

Fig. 4. Confusion matrix and performance evaluation metrics calculated from it, modified from Fawcett (2006).

Fig. 5. Schematic representation of the other three DCNN architectures we used. (A) ResNet v1152 architecture (He et al., 2016), (B) Inception v4 architecture (Szegedy et al., 2016), and (C) Inception ResNet v2 architecture (Szegedy et al., 2016). The ResNet block contains four types of residual blocks, each residual block stacks up its input (identity mapping) and output (residual mapping) to be the input of the next layer. The identity mappings can prevent the vanishing gradient issue. Each ResNet block implemented several times to decrease dimensions of the feature maps and increase the depth of the network. The stem module is a pre-processing step before the feature maps inputs to the Inception (ResNet) module, it contains two branches: convolutional and pooling branches. By combining two branches to significantly increase the channels for better feature extraction. The Inception module contains three types of Inception blocks, each module embeds a 
multiscale feature extractor, $1 \times 1,3 \times 3$, and $5 \times 5$ etc. convolutional layers. Each module was repeated several times to increase the depth of the network. The Inception ResNet module includes three kinds of modules, each module combined the characteristic of ResNet block and Inception module. The Inception ResNet modules decrease the channels and increase the depth of the architecture compared with modules from Inception v4. The Reduction module aims to reduce the dimensions to half, and increase the channels to make up for dimension decrease, it contains three or four branches and composed of one max pooling branch and two or three convolutional branches.

Fig. 6. Curves showing the evaluation of the (A) training loss, (B) training accuracy, (C) validation loss, and (D) validation accuracy with epoch for four DCNNs during the training processes. The hyper-parameter of these four DCNNs come from analysis number \#1, \#7, \#13, and \#19 in Table 2.

Fig. 7. Curves showing the evaluation of the (A) training loss, (B) training accuracy, (C) validation loss, and (D) validation accuracy with epoch during the training processes for Inception ResNet v2 with different hyper-parameter. The hyper-parameter of these four training models come from the analysis number $\# 20-\# 23$ in Table 2.

Fig. 8. Confusion matrix demonstrates true and predicted labels from the validation and test datasets, which tested on Inception ResNet v2 architecture.

Fig. 9. Visualization of the feature spatial distribution extracted by Inception ResNet v2 architecture from the 500 images (with $94.2 \%$ of accuracy) in the test dataset using t-SNE. There are 1536 features extracted from each test image after the last Inception ResNet v2 convolutional layer. Each number and colour quadrangle represent a class, the class order corresponding to alphabetical order 
in Table 1.

Table 1. Quantities of the training set, validation set, and test set for each fossil and abiotic grain group.

Table 2. The analysis results of four DCNN architectures. The network: $1=$ VGG-16; $2=$ ResNet $\mathrm{v} 1-152 ; 3=$ Inception $\mathrm{v} 4 ; 4$ = Inception ResNet v2. Load weights are load the pre-trained parameters from the ImageNet for variable initialize. Frozen layers are un-trainable layers. In the train layers column, all layers suggest that all layers of the architecture are trainable; half layers mean we trained the posterior half part of the architecture: for VGG-16 we trained conv4, conv5, cf6, fc7, and fc8 (Fig. 1A); for ResNet v1-152 we trained Block3, Block4, and the final layer (Fig. 5A); for Inception v4 we trained inception-B, Inception-C, and the final layer (Fig. 5B); for Inception ResNet v2 we trained Inception ResNet-B, Inception ResNet-C, and the final layer (Fig. 5C). Drop out range from $0^{-1}$, where 0 stands for probabilistically removing or "dropping out" all the inputs to the next layer, 1 stands for no dropping out. Decay step stands for each corresponding iteration, the learning rate will multiply the decay rate. Batch nor. $=$ Batch normalization. This function aims to scale and standardize the input training data before feeding it to a model for training and benefit to model convergence. Num aug. $=$ Number of methods for data augmentation. For VGG and ResNet, 3 means no crop of training images (i.e., methods (1), (3), and (5) in section 3.3); 4 means randomly crop training images; For Inception networks, 4 means no crop of training images (i.e., methods (1), (3), (5), and (6) in section 3.3), and 5 means randomly crop training images. Optimizer is the algorithm or method used to optimize the performance of the neural networks by providing the 
appropriate optimization direction. 


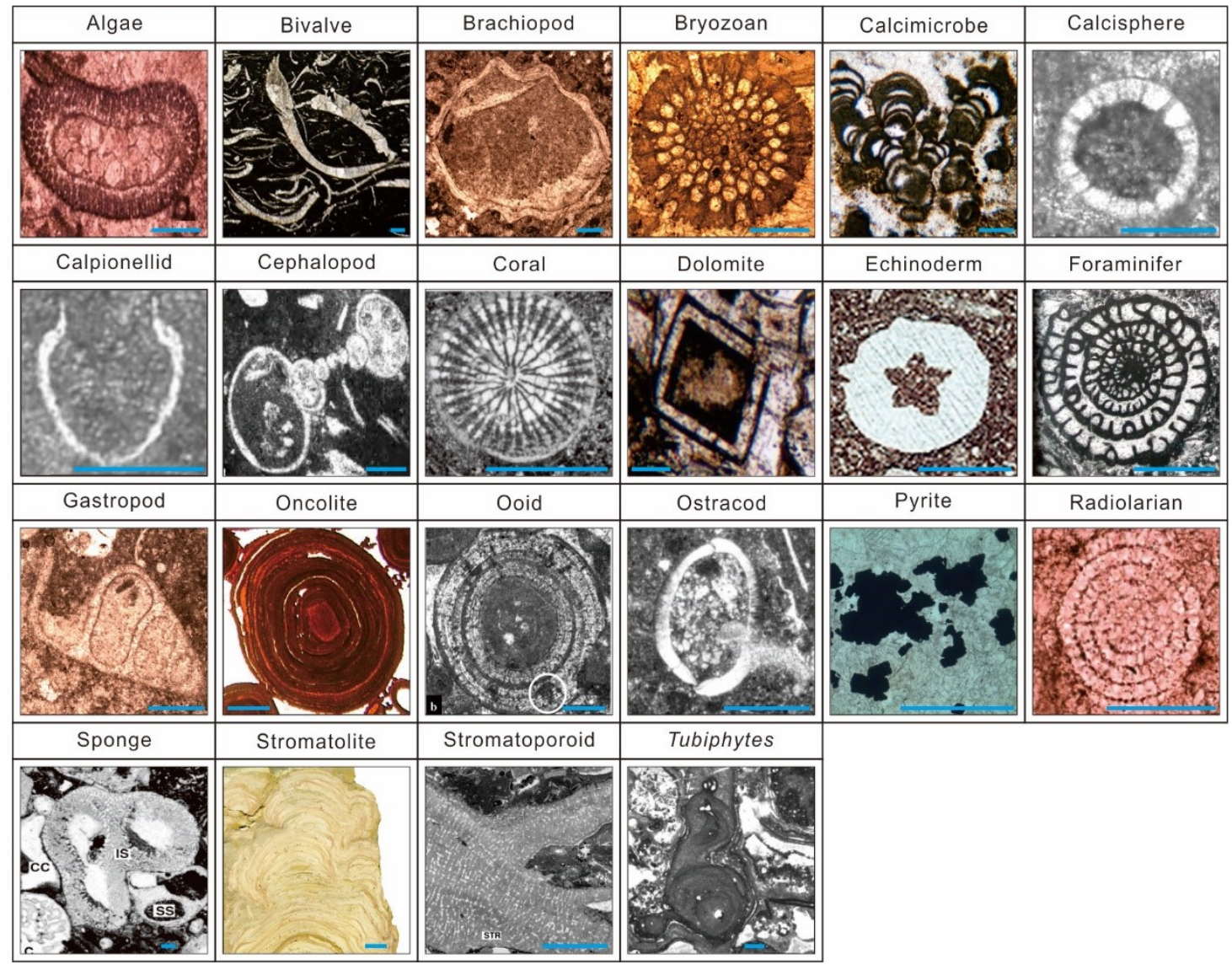

Fig. 1 


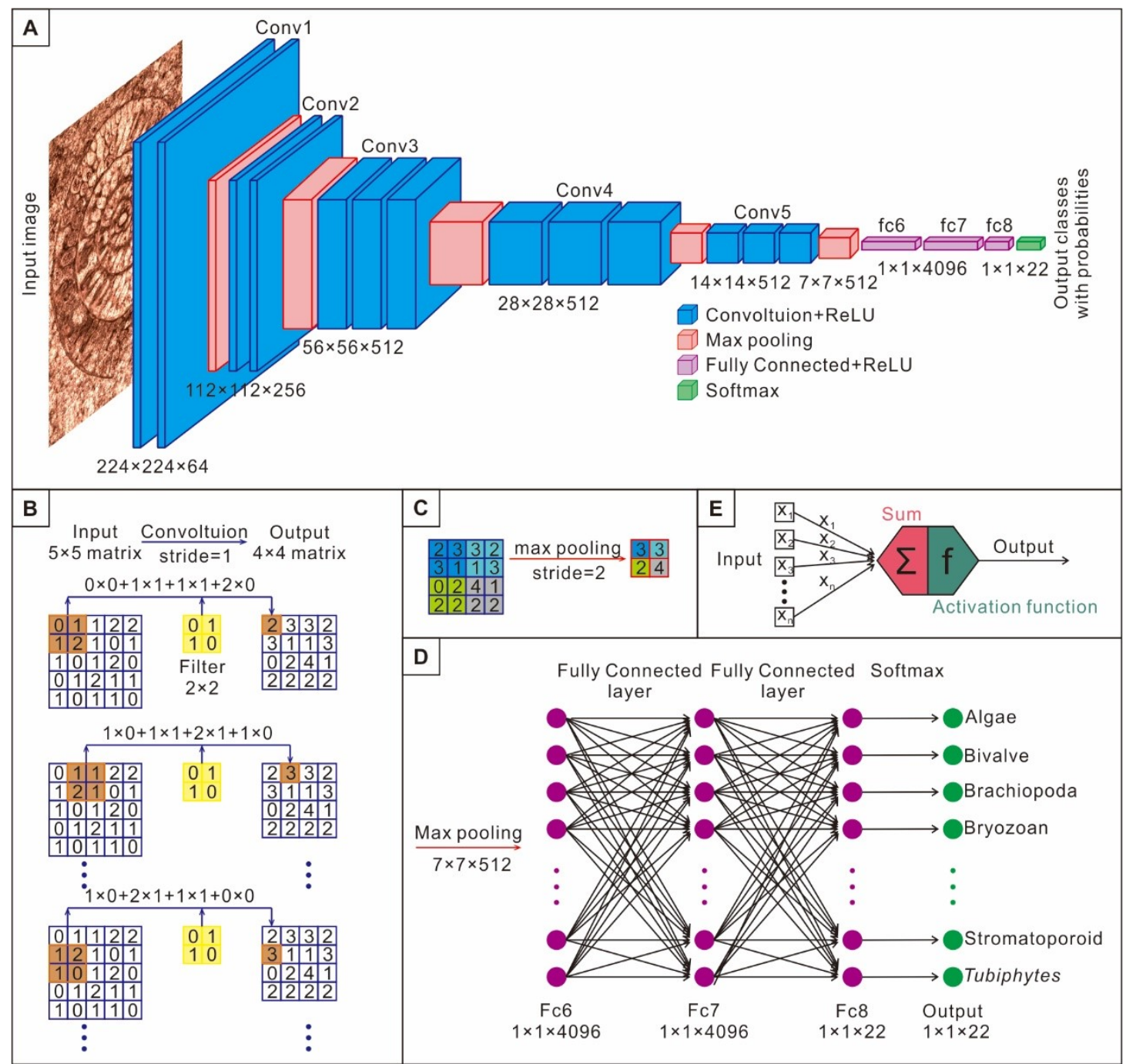

Fig. 2 


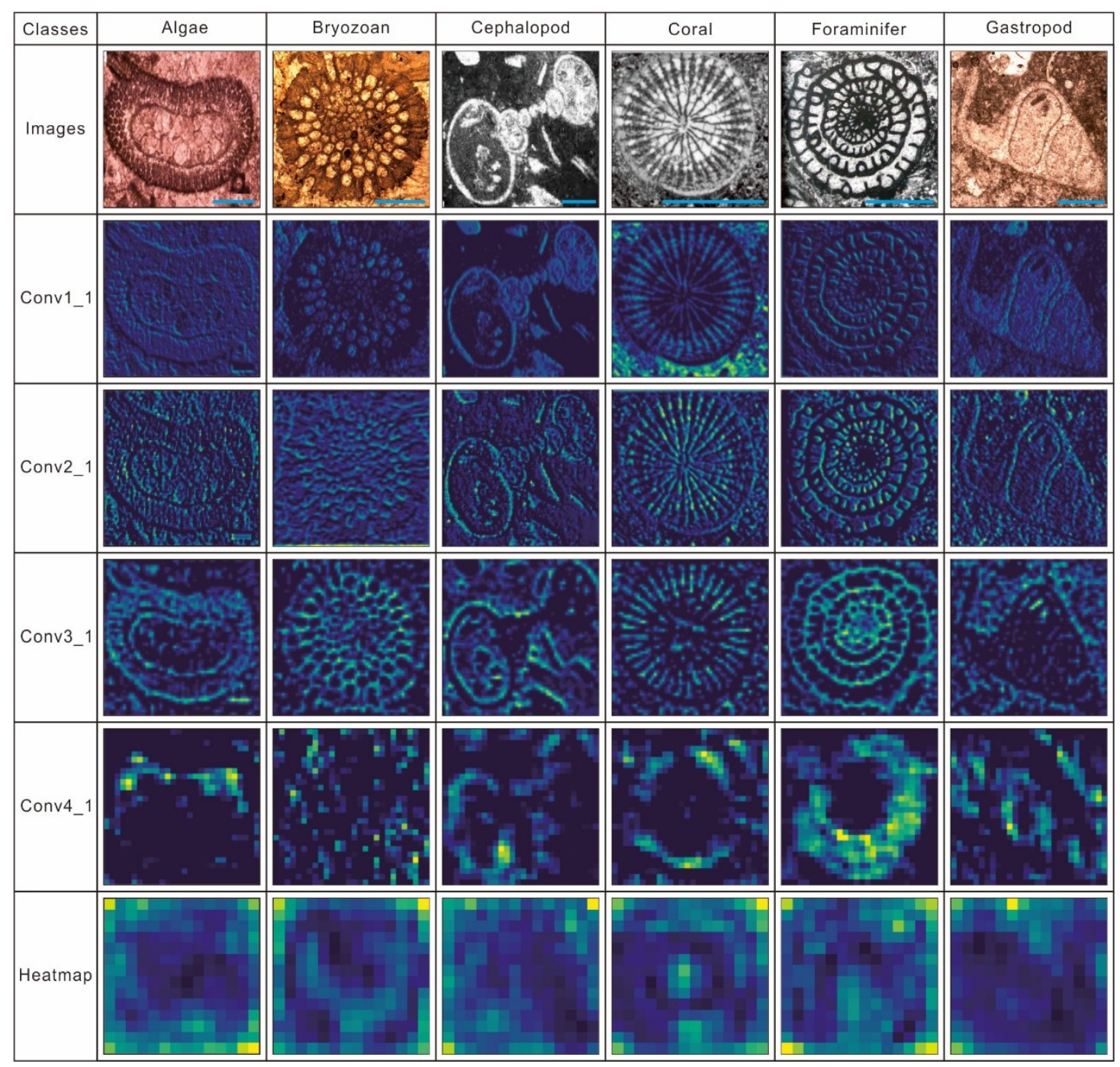

Fig. 3 


\begin{tabular}{|c|c|c|c|c|}
\hline \multirow{2}{*}{\multicolumn{2}{|c|}{$\begin{array}{c}\text { Confusion } \\
\text { matrix }\end{array}$}} & \multicolumn{2}{|c|}{ True labels } & \multirow{3}{*}{$\begin{array}{l}\text { Evaluation } \\
\text { Precision } \\
\frac{T P}{(T P+F P)}\end{array}$} \\
\hline & & \multirow{2}{*}{\begin{tabular}{|c|} 
Positive \\
$\begin{array}{c}\text { True } \\
\text { Positive }\end{array}$
\end{tabular}} & \multirow{2}{*}{\begin{tabular}{|c|} 
Negative \\
\\
False \\
Positive
\end{tabular}} & \\
\hline $\begin{array}{l}\frac{\infty}{10} \\
\frac{0}{0}\end{array}$ & 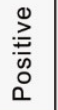 & & & \\
\hline $\begin{array}{l}\frac{ \pm}{0} \\
\frac{0}{0} \\
\frac{\Phi}{0}\end{array}$ & 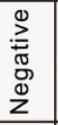 & $\begin{array}{c}\text { False } \\
\text { Negative }\end{array}$ & $\begin{array}{c}\text { True } \\
\text { Negative }\end{array}$ & $\begin{array}{c}\text { F1 score } \\
\frac{2^{*} \text { Prescision }^{*} \text { Recall }}{(\text { Prescision }+ \text { Recall })}\end{array}$ \\
\hline \multicolumn{2}{|c|}{ Evaluation } & \multicolumn{2}{|c|}{$\begin{array}{l}\text { Recall } \\
\frac{T P}{(T P+F N)}\end{array}$} & $\begin{array}{c}\text { Accuracy } \\
\frac{T P+T N}{(T P+T N+F P+F N)}\end{array}$ \\
\hline
\end{tabular}

Fig. 4 


\begin{tabular}{|c|}
\hline Input image \\
\hline$\sqrt{2} 224 \times 224 \times 3$ \\
\hline Conv1
\end{tabular}

Conv 1

\勺 $112 \times 112 \times 64$

Max pooling

$\checkmark 56 \times 56 \times 64$

$3 \times$ ResNet Block-1

$\sqrt{56 \times 56 \times 256}$

$8 \times$ ResNet Block-2

प28×28×512

$36 \times$ ResNet Block-3

\勺 $14 \times 14 \times 1024$

$3 \times$ ResNet Block-4

यु $7 \times 7 \times 2048$

Average pooling

$\checkmark 2048$

Drop out

$\checkmark 22$

Softmax
B

\begin{tabular}{|c|}
\hline Input image \\
\hline$\bigvee 299 \times 299 \times 3$ \\
\hline Stem
\end{tabular}

Stem

\{ $35 \times 35 \times 384$

$4 \times$ Inception-A

रु $35 \times 35 \times 384$

Reduction-A

V $17 \times 17 \times 1024$

$7 \times$ Inception-B

$\checkmark 17 \times 17 \times 1024$

Reduction-B

\y $8 \times 8 \times 1356$

$3 \times$ Inception-C

रु $8 \times 8 \times 1536$

Average pooling

\1536

Drop out

प22

Softmax
C

Input image

V $299 \times 299 \times 3$

Stem

V $35 \times 35 \times 256$

$5 \times$ Inception ResNet-A v $35 \times 35 \times 256$

Reduction-A

$\checkmark$ 17×17×896

$10 \times$ Inception ResNet-B

V $17 \times 17 \times 896$

Reduction-B

$\checkmark 8 \times 8 \times 1792$

$5 \times$ Inception ResNet-C

\y $8 \times 8 \times 1792$

Average pooling

v1792

\}22

Softmax

Fig. 5 

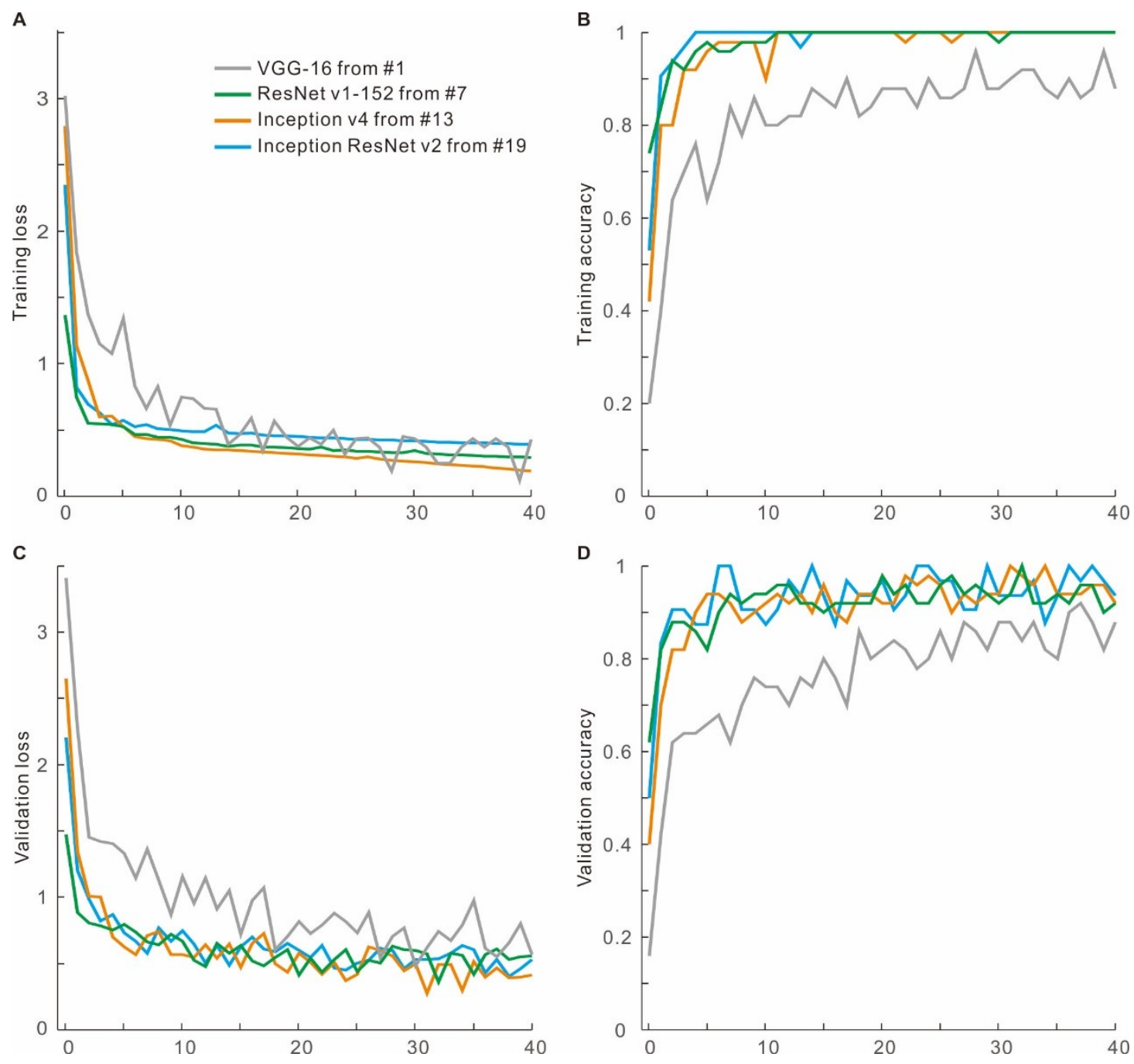

Fig. 6 

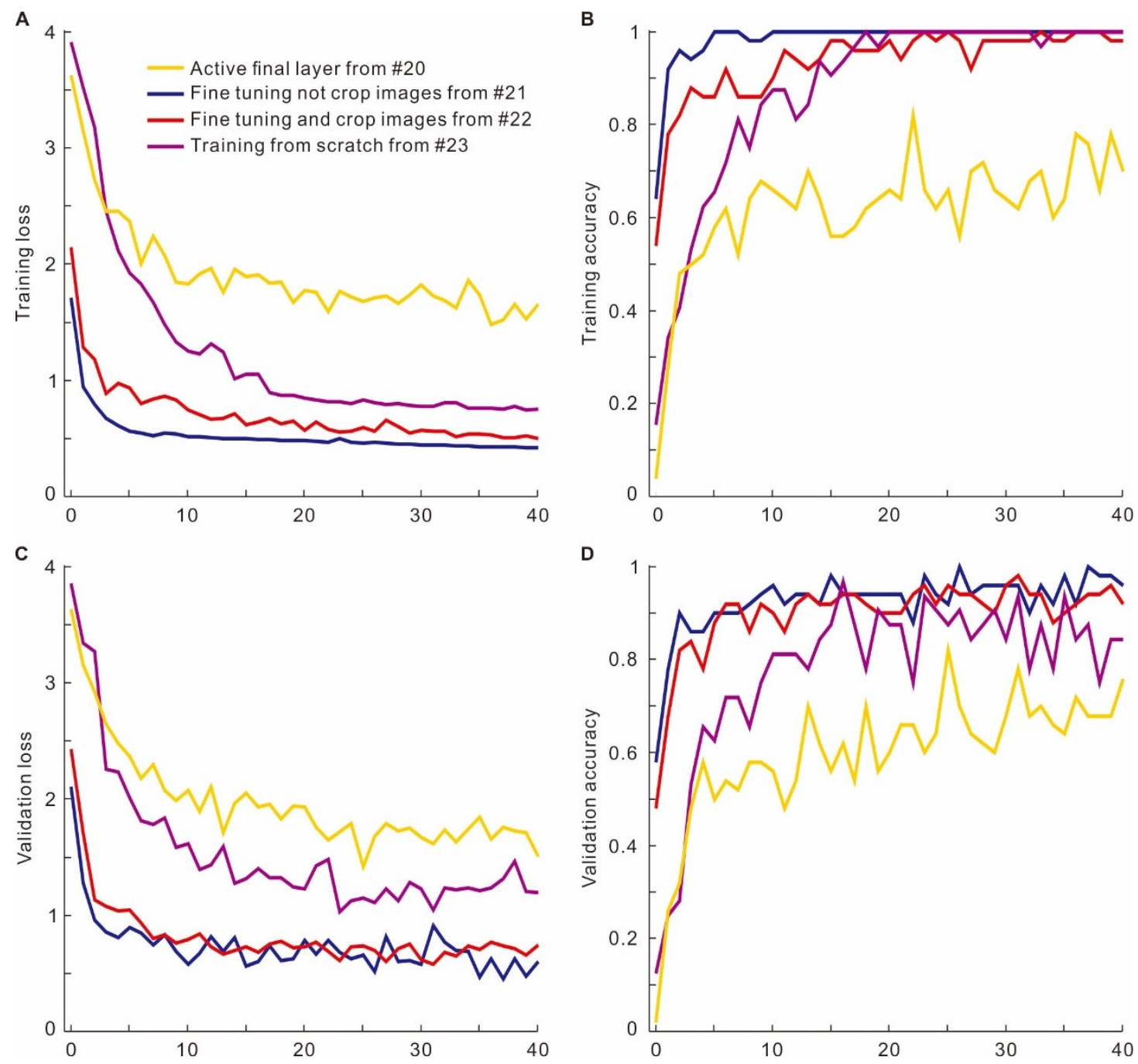

Fig. 7 


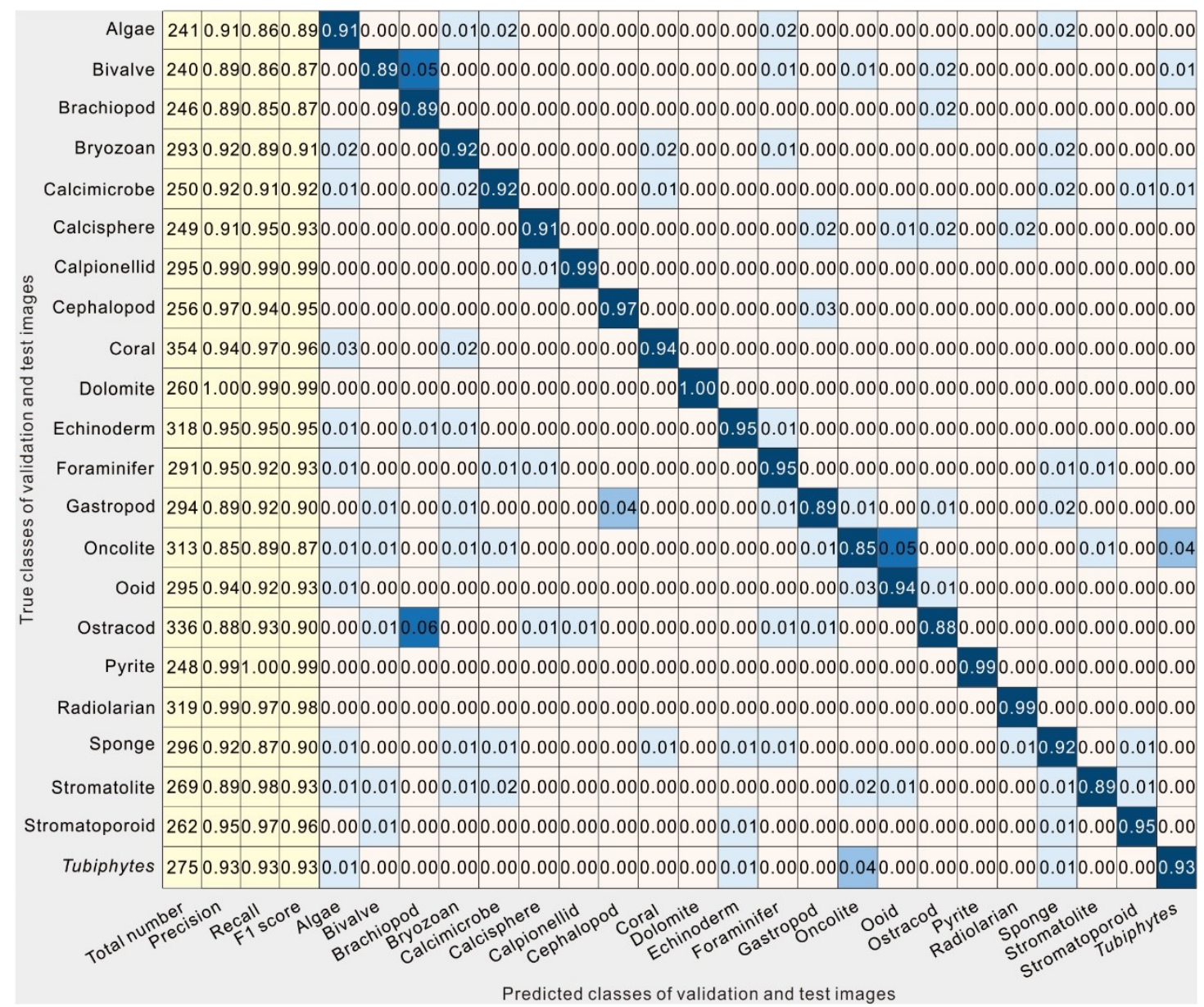

Fig. 8 
Visualize last layer with $94.20 \%$ accuracy

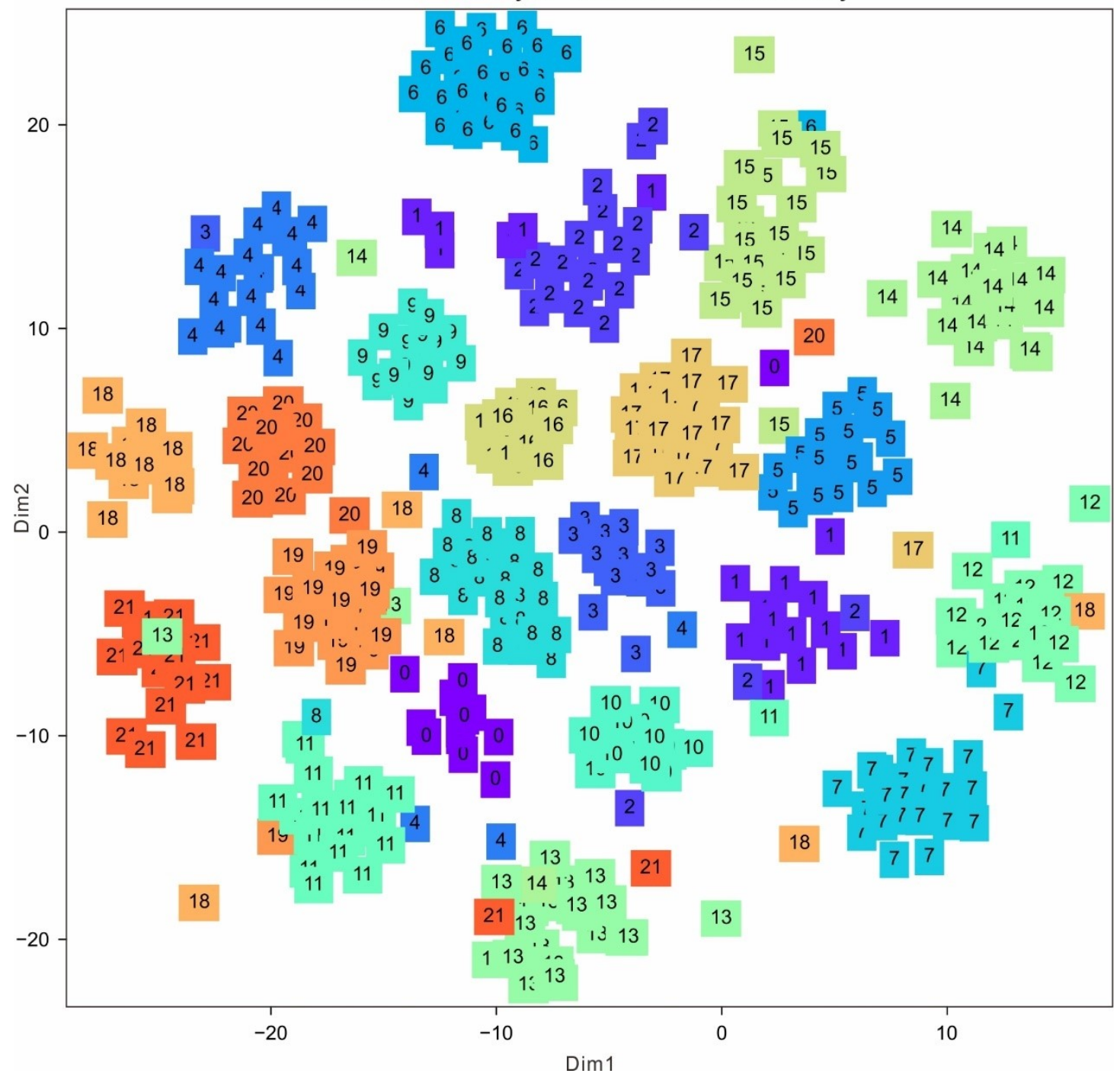

Fig. 9 


\begin{tabular}{|c|c|c|c|c|c|}
\hline Order & Classes & $\begin{array}{c}\text { Training } \\
\text { set }(80 \%)\end{array}$ & $\begin{array}{l}\text { Validation } \\
\text { set }(15 \%)\end{array}$ & $\begin{array}{c}\text { Test set } \\
(5 \%)\end{array}$ & Total \\
\hline 0 & Algae & 1037 & 195 & 64 & 1296 \\
\hline 1 & Bivalve & 993 & 186 & 62 & 1241 \\
\hline 2 & Brachiopod & 1011 & 189 & 63 & 1263 \\
\hline 3 & Bryozoan & 1162 & 218 & 72 & 1452 \\
\hline 4 & Calcimicrobe & 1036 & 194 & 64 & 1294 \\
\hline 5 & Calcisphere & 982 & 184 & 61 & 1227 \\
\hline 6 & Calpionellid & 1129 & 212 & 70 & 1411 \\
\hline 7 & Cephalopod & 1039 & 195 & 64 & 1298 \\
\hline 8 & Coral & 1317 & 247 & 82 & 1646 \\
\hline 9 & Dolomite & 1023 & 192 & 63 & 1278 \\
\hline 10 & Echinoderm & 1260 & 237 & 78 & 1575 \\
\hline 11 & Foraminifer & 1260 & 236 & 78 & 1574 \\
\hline 12 & Gastropod & 1135 & 213 & 70 & 1418 \\
\hline 13 & Oncolite & 1218 & 228 & 76 & 1522 \\
\hline 14 & Ooid & 1168 & 219 & 73 & 1460 \\
\hline 15 & Ostracod & 1288 & 242 & 80 & 1610 \\
\hline 16 & Pyrite & 996 & 186 & 62 & 1244 \\
\hline 17 & Radiolarian & 1259 & 236 & 78 & 1573 \\
\hline 18 & Sponge & 1223 & 229 & 76 & 1528 \\
\hline 19 & Stromatolite & 1025 & 192 & 64 & 1281 \\
\hline 20 & Stromatoporoid & 998 & 187 & 62 & 1247 \\
\hline \multirow[t]{2}{*}{21} & Tubiphytes & 1102 & 207 & 68 & 1377 \\
\hline & Total & 24661 & 4624 & 1530 & 30815 \\
\hline
\end{tabular}

Table 1 


\begin{tabular}{|c|c|c|c|c|c|c|c|c|c|c|c|c|c|c|c|c|c|c|c|}
\hline Order & Network & $\begin{array}{l}\text { Batch } \\
\text { size }\end{array}$ & $\begin{array}{c}\text { Load } \\
\text { weights }\end{array}$ & $\begin{array}{l}\text { Frozen } \\
\text { layers }\end{array}$ & Train layers & $\begin{array}{l}\text { Drop } \\
\text { out }\end{array}$ & $\begin{array}{c}\text { Start } \\
\text { learning rate }\end{array}$ & $\begin{array}{l}\text { Decay } \\
\text { step }\end{array}$ & $\begin{array}{c}\text { Decay } \\
\text { rate }\end{array}$ & $\begin{array}{l}\text { Batch } \\
\text { nor. }\end{array}$ & $\begin{array}{l}\text { Num } \\
\text { aug. }\end{array}$ & Optimizer & $\begin{array}{c}\text { Epoch } \\
\text { ran }\end{array}$ & $\begin{array}{c}\text { Max } \\
\text { tra } \\
\text { acc }\end{array}$ & $\begin{array}{l}\text { Min } \\
\text { tra } \\
\text { loss }\end{array}$ & $\begin{array}{l}\text { Max } \\
\text { val } \\
\text { acc }\end{array}$ & $\begin{array}{l}\text { Min } \\
\text { val } \\
\text { loss }\end{array}$ & $\begin{array}{c}\text { Max top } 1 \\
\text { test acc }\end{array}$ & $\begin{array}{c}\text { Max top } 3 \\
\text { test acc }\end{array}$ \\
\hline 1 & 1 & 50 & Yes & Yes & Half layers & 0.8 & 0.0001 & 400 & 0.96 & Yes & 3 & Adam & 50 & 0.96 & 0.12 & 0.84 & 0.52 & 0.91 & 0.98 \\
\hline 2 & 1 & 50 & Yes & Yes & Half layers & 0.8 & 0.00001 & 1000 & 0.96 & Yes & 3 & Adam & 50 & 0.96 & 0.15 & 0.84 & 0.66 & 0.88 & 0.96 \\
\hline 3 & 1 & 50 & Yes & No & All layers & 0.8 & 0.0001 & 400 & 0.96 & No & 4 & Adam & 50 & 1.00 & 0.43 & 0.94 & 0.61 & 0.88 & 0.97 \\
\hline 4 & 1 & 100 & Yes & Yes & Half layers & 0.8 & 0.0001 & 400 & 0.96 & Yes & 3 & Adam & 40 & 1.00 & 0.00 & 0.91 & 0.48 & 0.86 & 0.97 \\
\hline 5 & 1 & 50 & No & No & All layers & 0.8 & 0.0001 & No & & No & 3 & Adam & 60 & 1.00 & 0.55 & 0.72 & 1.65 & 0.59 & 0.71 \\
\hline 6 & 1 & 50 & Yes & Yes & Half layers & 0.5 & 0.0001 & 400 & 0.96 & No & 3 & Adam & 50 & 1.00 & 0.78 & 0.96 & 0.91 & 0.91 & 0.98 \\
\hline 7 & 2 & 50 & Yes & Yes & Half layers & 1.0 & 0.0001 & 400 & 0.96 & Yes & 3 & Adam & 40 & 1.00 & 0.29 & 1.00 & 0.36 & 0.93 & 0.99 \\
\hline 8 & 2 & 50 & Yes & Yes & Half layers & 0.8 & 0.0001 & 400 & 0.96 & Yes & 4 & RMSP & 40 & 1.00 & 0.38 & 0.98 & 0.49 & 0.91 & 0.98 \\
\hline 9 & 2 & 50 & Yes & Yes & All layers & 0.8 & 0.0001 & 400 & 0.96 & Yes & 3 & Adam & 40 & 1.00 & 0.27 & 0.98 & 0.35 & 0.91 & 0.99 \\
\hline 10 & 2 & 50 & Yes & Yes & Half layers & 1.0 & 0.001 & 300 & 0.96 & Yes & 3 & Adam & 40 & 1.00 & 0.19 & 0.96 & 0.46 & 0.89 & 0.98 \\
\hline 11 & 2 & 50 & No & No & All layers & 0.8 & 0.0001 & 500 & 0.96 & Yes & 3 & Adam & 60 & 1.00 & 2.39 & 0.82 & 3.50 & 0.68 & 0.86 \\
\hline 12 & 2 & 64 & Yes & Yes & Half layers & 0.8 & 0.0001 & 400 & 0.96 & Yes & 3 & Adam & 40 & 1.00 & 0.10 & 0.95 & 0.34 & 0.90 & 0.97 \\
\hline 13 & 3 & 50 & Yes & Yes & Half layers & 0.8 & 0.0001 & 400 & 0.96 & Yes & 4 & RMSP & 40 & 1.00 & 0.19 & 1.00 & 0.28 & 0.94 & 0.99 \\
\hline 14 & 3 & 50 & Yes & No & All layers & 0.8 & 0.0001 & 400 & 0.96 & Yes & 4 & RMSP & 40 & 1.00 & 0.26 & 0.98 & 0.37 & 0.94 & 0.99 \\
\hline 15 & 3 & 50 & Yes & Yes & Half layers & 0.5 & 0.0001 & 400 & 0.96 & Yes & 4 & RMSP & 40 & 1.00 & 0.31 & 0.98 & 0.44 & 0.93 & 0.99 \\
\hline 16 & 3 & 50 & Yes & Yes & Half layers & 0.8 & 0.0001 & No & & Yes & 5 & RMSP & 40 & 1.00 & 0.52 & 0.96 & 0.54 & 0.89 & 0.98 \\
\hline 17 & 3 & 32 & Yes & Yes & Half layers & 0.8 & 0.00001 & 1000 & 0.96 & Yes & 5 & RMSP & 40 & 0.94 & 0.77 & 0.94 & 0.60 & 0.82 & 0.95 \\
\hline 18 & 3 & 50 & No & No & All layers & 0.8 & 0.0001 & 400 & 0.96 & Yes & 4 & RMSP & 60 & 1.00 & 1.12 & 0.90 & 1.75 & 0.73 & 0.90 \\
\hline 19 & 4 & 32 & Yes & Yes & All layers & 0.8 & 0.0001 & 400 & 0.96 & Yes & 4 & Adam & 40 & 1.00 & 0.39 & 1.00 & 0.40 & 0.95 & 0.99 \\
\hline 20 & 4 & 50 & Yes & Yes & Last layer & 0.8 & 0.0001 & 400 & 0.96 & Yes & 4 & Adam & 40 & 0.82 & 1.49 & 0.82 & 1.42 & 0.67 & 0.86 \\
\hline 21 & 4 & 50 & Yes & Yes & Half layers & 0.8 & 0.0001 & 400 & 0.96 & Yes & 4 & Adam & 40 & 1.00 & 0.42 & 1.00 & 0.45 & 0.94 & 0.99 \\
\hline 22 & 4 & 50 & Yes & Yes & Half layers & 0.8 & 0.0001 & 400 & 0.96 & Yes & 5 & Adam & 40 & 1.00 & 0.50 & 0.98 & 0.58 & 0.93 & 0.99 \\
\hline 23 & 4 & 32 & No & No & All layers & 0.8 & 0.0001 & 400 & 0.96 & Yes & 4 & Adam & 60 & 1.00 & 0.71 & 0.97 & 0.83 & 0.82 & 0.95 \\
\hline 24 & 4 & 100 & Yes & Yes & Half layers & 0.8 & 0.0001 & 400 & 0.96 & Yes & 4 & Adam & 40 & 1.00 & 0.00 & 0.96 & 0.08 & 0.94 & 0.99 \\
\hline
\end{tabular}

Table 2

$52 / 52$ 